\title{
Arthroscopic Shaver Design Parameters Controlled Laboratory Testing
}

\author{
Nader Hafez ${ }^{1}$, Mostafa R. A. Atia ${ }^{2}$, Amr A. Elfeky ${ }^{3}$, Mohamed I. El-Anwar ${ }^{3 *}$ \\ ${ }^{1}$ Department ofMechanical Engineering, Canadian International College, Cairo, Egypt; ${ }^{2}$ Department of Mechanical Engineering, \\ Arab Academy for Science and Technology, Cairo, Egypt; ${ }^{3}$ Department of Mechanical Engineering, National Research Centre, \\ Cairo, Egypt
}

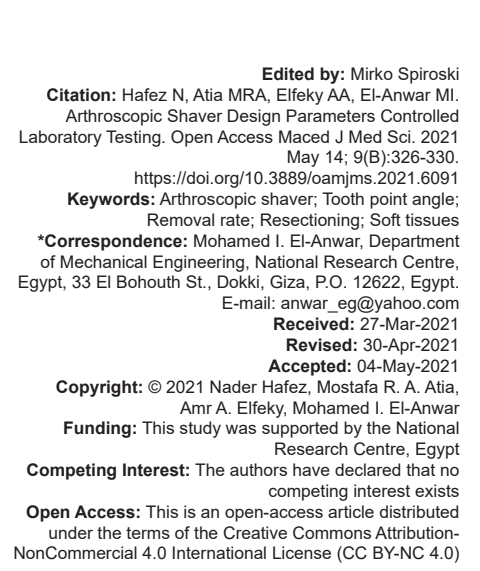

\section{Abstract}

AIM: Arthroscopic shaver is a widely used surgical single-use cutting tools. Its life and usability are important parameter for operation quality. In this research, arthroscopic shaver design and operation parameters are shot.

METHODS: The relations between the cutting variables and output parameters are studied to achieve the recommended design and cutting conditions. The interesting variables are the tooth point angle, cutting speed, and contact force. Other variables are maintained constant within the range of commercial shavers. The interesting cutting parameters are stresses on shaver tooth, resection rate, and damage experienced by the teeth. The cut quality is assessed based on the expertise medical opinion. Special setup is designed and prepared to test re-sectioning of soft tissues under different operating conditions.

RESULTS: The experimental results show that the cutting tooth angle between $55^{\circ}$ to $62^{\circ}$ achieved higher resection (material removal) rates while experiencing less damage and teeth blunting. Additionally, the cutting speed of $14 \mathrm{~m} /$ min increases the material resection by $60 \%$ than the lower ones. Where, the resection performance decreases by approximately $50 \%$ every consecutive three minutes test run.

CONCLUSION: These results agree with the previous theoretical work using finite element modeling and simulation

\section{Introduction}

Arthroscopic surgery's development is seen as a turning point in orthopedic surgery. The origin of the term arthroscopy comes from the Greek words arthros and scopein, which mean joint and look, respectively.

Recently, the arthroscopic surgery is the most important developments in orthopedic surgery. Arthroscopic surgery is considered to be a minimal invasive surgery that provides a limit need of incisions size, less time of wound healing, and decreases the risk of infection, which allows higher degree of clinical accuracy with low morbidity that provides a controlled management of the soft tissue and bony resections as part of reconstructive arthroscopic procedures. Powered cutter arthroscopic shaver system plays an important role in arthroscopic surgeries. They are designed for use in different clinical situations and for specific functions to cut massive tissue in a short time. However, the principles of the design remain similar (Figure 1).

The shaver blade consists of an outer hollow sheath and an inner hollow rotating cannula with corresponding windows for suction and cutting; a "tube within a tube" mechanism [1]. The window of the inner sheath functions as a two-edged cylindrical blade that spins within the outer hollow tube [2]. The shape and geometry of the edges of the inner and outer tube tips determine the degree of aggression of the blade. They are available in shaft diameters. They are broadly divided according to the type of tissue cutting [3].

\section{Blades cut soft tissue}

The oscillation mode at lower speeds of range 188-1200 rpm is a better mode for cutting soft tissue [4]. The level of aggressiveness can be controlled according to three designs as shown in Figure 2. Smooth inner tube and smooth outer tube as shown in Figure 2 a represent the lowest level of aggressiveness. Smooth outer tube with toothed inner tube shown in Figure $2 \mathrm{~b}$ represents a medium level of aggressiveness. Figure 2c shows the toothed inner and outer tubes which represents the highest level of aggressiveness.

\section{Blades cut bony resection}

The forward mode at speed 5000 rpm was found to be the ideal performance for cutting bony resection [4]. 


\section{Blades cut bony and soft-tissue resection}

Inner tube with helical shape allow cutting of bone, soft tissue, and periosteum without clogging. Extra coating may be added to reduce blade friction and allow smooth cutting for both soft tissue and bone [4].

The most frequently performed arthroscopy in the knee is a meniscectomy, which usually consists of cutting a stable rim in a ruptured meniscus to prevent further tearing [5]. The access portals are routinely placed at the anterior side of the joint level medially and laterally from the patella tendon [5], as presented in Figure 1. The combination of these limited access points, the complex curvature of the condyles, and the location of the lesions in the meniscus cause difficulties in treatment of the meniscus.

Dried blood, saline, and other deposits inside the hand-pieces can be a major cause of equipment malfunction or infection unless cleaned and sterilized regularly and adequately. Most shaver blades now on the market are for single use only [4].

Aiming to enhance designs for arthroscopic shavers and implants used in joint surgery, this study is concerned with the arthroscopic shaver's design, cutting simulation, and experimental verification of the simulation results. Cutting simulation was presented in previous publications [6], [7], the experimental

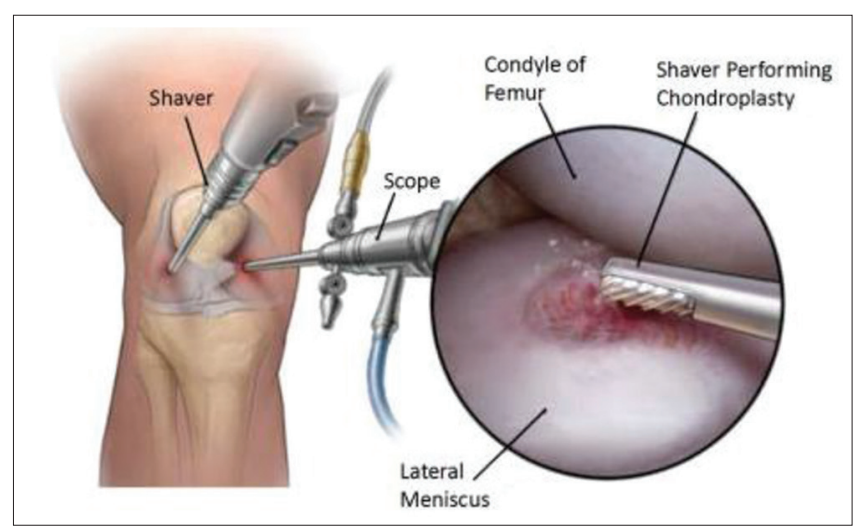

Figure 1: Arthroscopic minimally invasive surgery [1] (laboratory tests) part only is presented within this article.

\section{Materials and Methods}

Three shaver designs with different teeth point angles; $55^{\circ}, 62^{\circ}$, and $70^{\circ}$; are manufactured from stainless steel 304 with two diameters $4 \mathrm{~mm}$ and $6 \mathrm{~mm}$. Then, experiments are conducted to test the designed and manufactured shavers to compare their performance with the commercial ones. Qualitative analysis using microscopic images is presented as well as quantitative analysis for the resection (material removal) rate. Validity of the finite element (FE) model results [6], [7], is discussed in light of the conducted experimental work.

Figure 3 presents the designed test rig, which is constructed in the biomechanics laboratory at National Research Centre. Figure 4 shows the control diagram and components, where a servomotor of $0.4 \mathrm{~kW}$ was driven by high-performance servo driver to maximum $1.27 \mathrm{Nm}$ torque and $3000 \mathrm{rpm}$. WPL soft software was used in programming the servo driver. It manages the

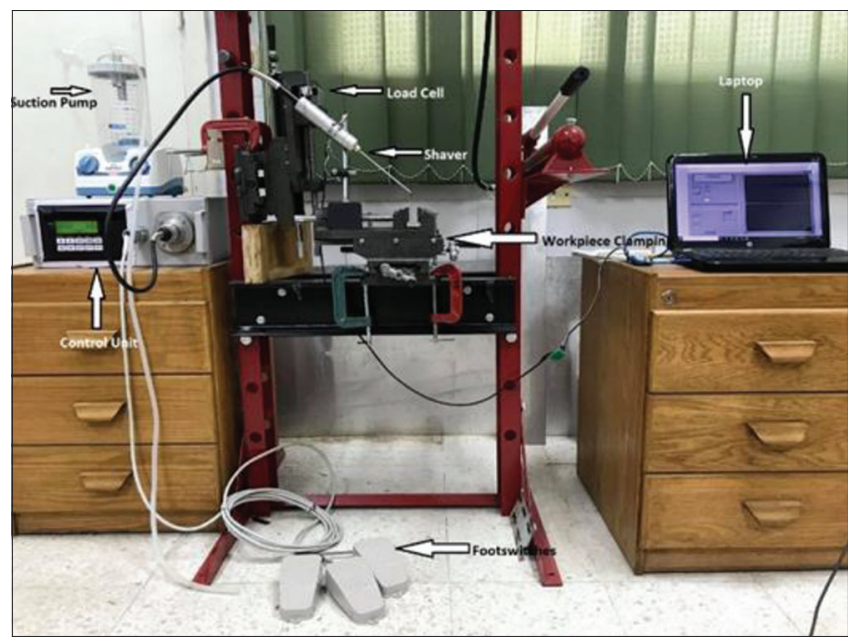

Figure 3: Laboratory set-up for experiments

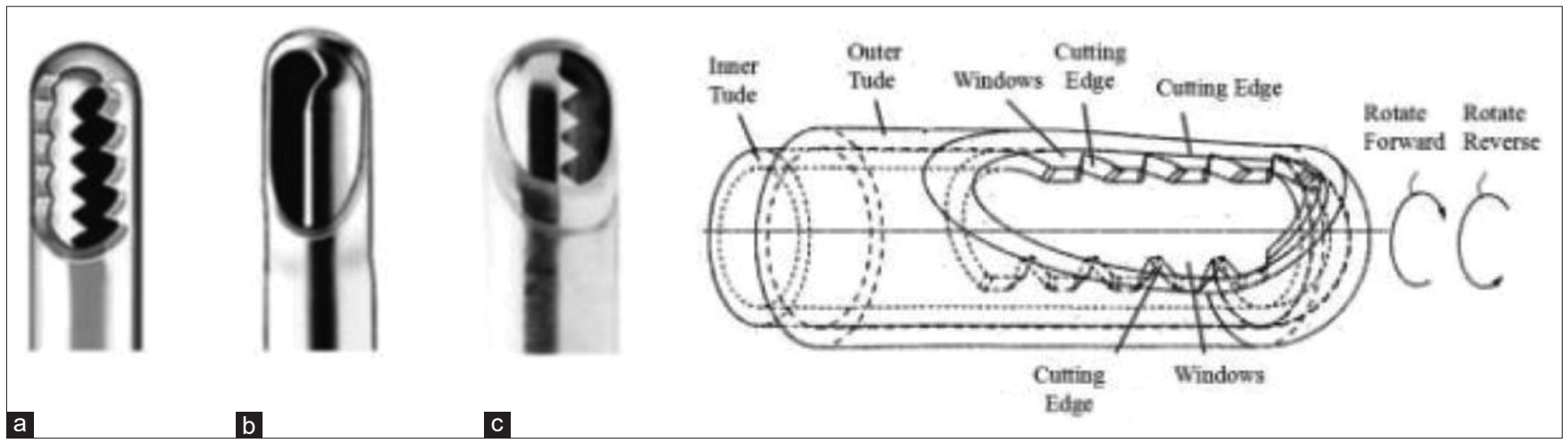

Figure 2: Various types of shaver blades design [1] 


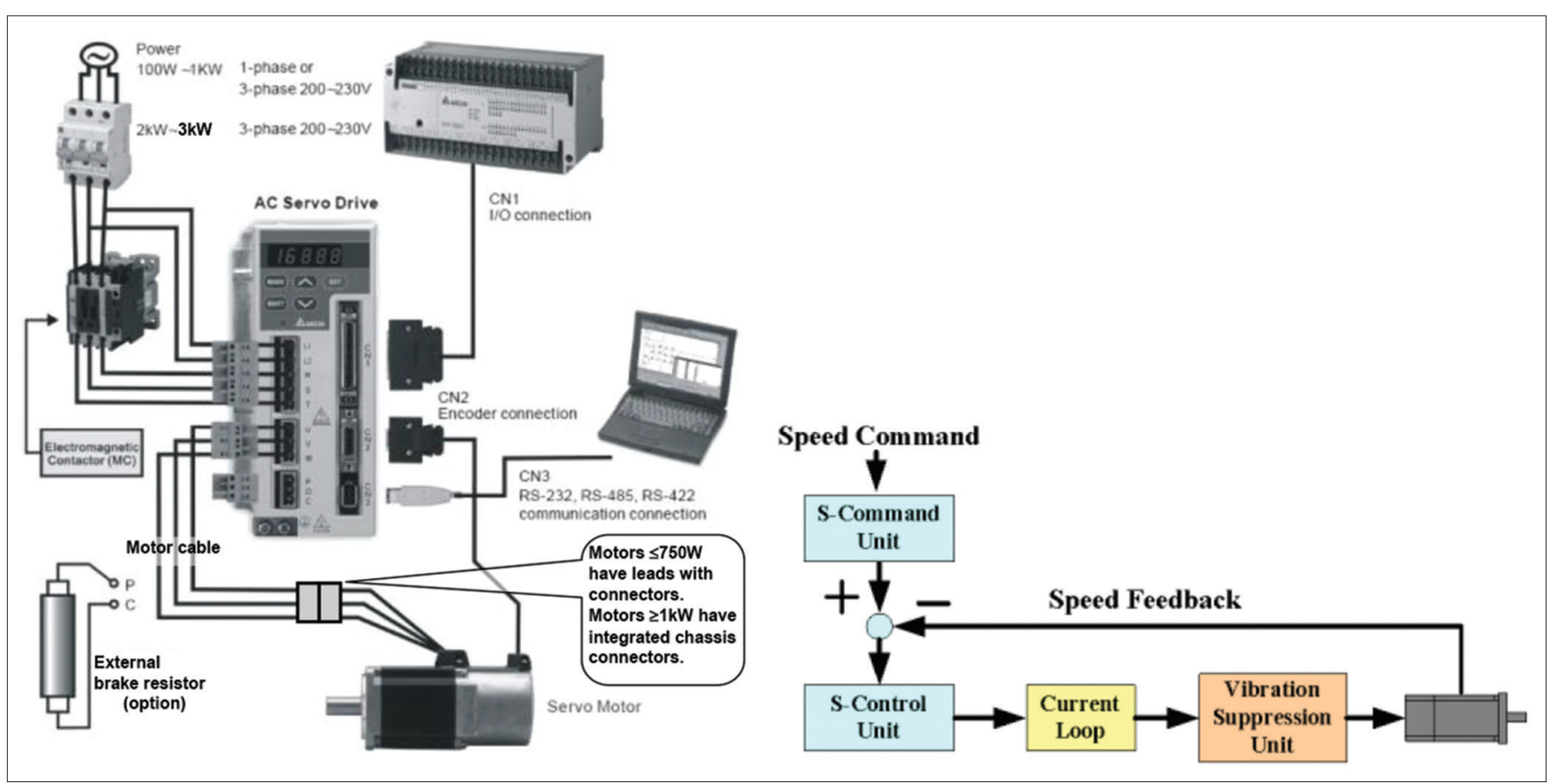

Figure 4: Control unit components and control block diagram

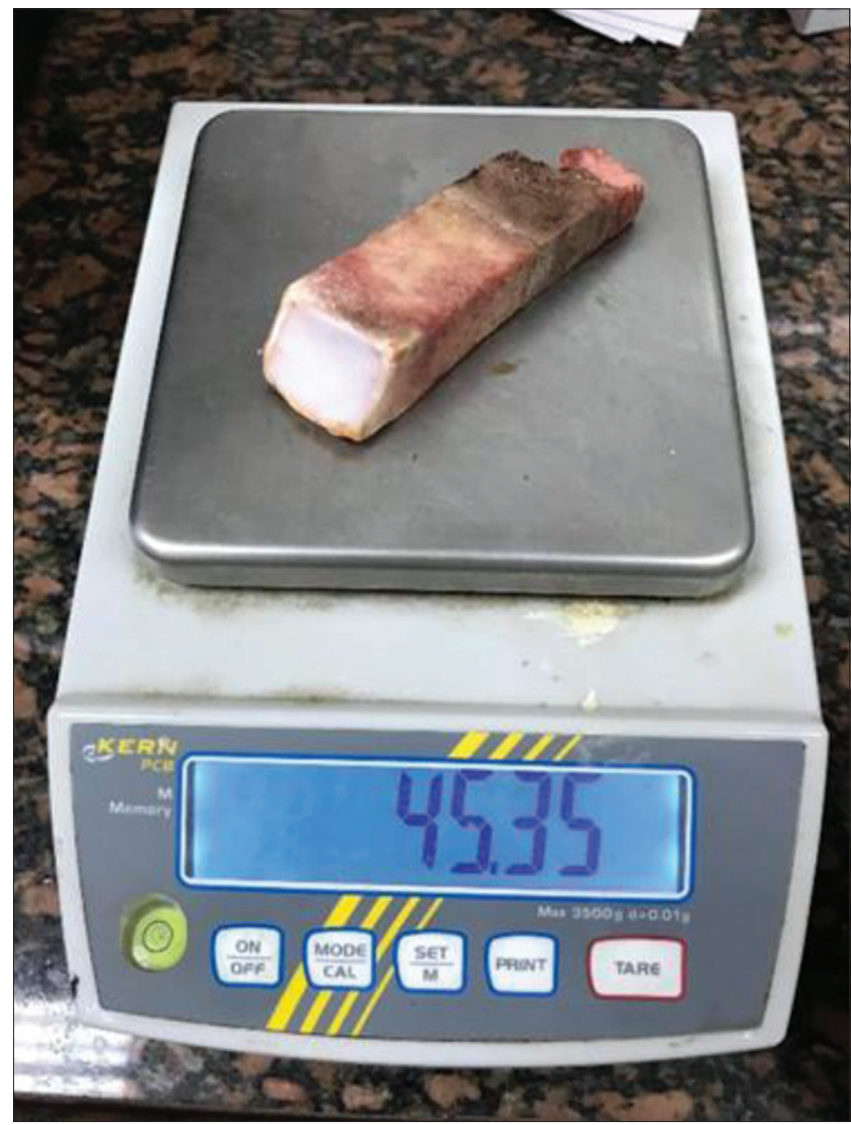

Figure 5: Weighing test specimen

shaver's rotational speed and cutting direction through footswitches in continuous and reciprocating modes.

The shaver is supported by specially designed and manufactured mechanism, which applies a contact force similar to that applied by the surgeon. The mechanism has a $2 \mathrm{~kg}$ load cell (HX711 Weighing Sensor Module) [8] to measure the contact force during the tests. A suction pump, New Aspiret suction pump, $15 \mathrm{lit} / \mathrm{min} 0.75$ bar [9], is connected to the shaver holder to clean the debris of cutting and any lubricating fluids used during experiment (saline 5\%). Test specimens are prepared from fresh bovine cartilage, using commercial bovine joints that are cut by a saw to the proper size suitable for experiments.

Using a KERN precision scale [10] with precision of $0.01 \mathrm{~g}$ (Figure 5), every specimen is weighted before and every 3 min of shaving. Microscopic images are taken for the shaver's teeth before and after $12 \mathrm{~min}$ of cutting for comparison. Figure 6a shows the shaver during cutting test. Figure $6 \mathrm{~b}$ and $\mathrm{c}$ shows example for cutting teeth images before and after testing.

Results of contact force, material removal, and microscopic images of the shaver blades are obtained, analyzed, and the overall performance is compared to that obtained from the previous simulation work.

\section{Results}

A total of 96 experiments are conducted. A test is carried out for each shaver diameter (4 and $6 \mathrm{~mm}$ ) and tooth point angle $\left(55^{\circ}, 62^{\circ}\right.$, and $\left.70^{\circ}\right)$. For every design, four cutting speeds $(1200,1500,1800$, and

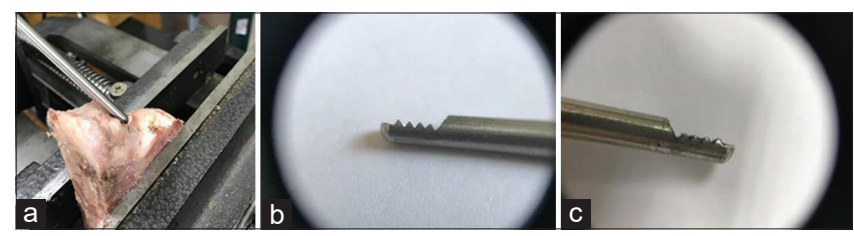

Figure 6: (a) Shaver with $55^{\circ}$ point angle during test and sample images, (b) before and (c) after testing 
2100 RPM) were tested under pressing load of $5 \mathrm{~N}$. Every specimen is weighted before and after every 3 min (reading) with total of 12 min (four readings) of cutting. Figures 7 and 8 compare the removal material weight every $3 \mathrm{~min}$ (in $\mathrm{gm}$ ) to extract finding of this study.

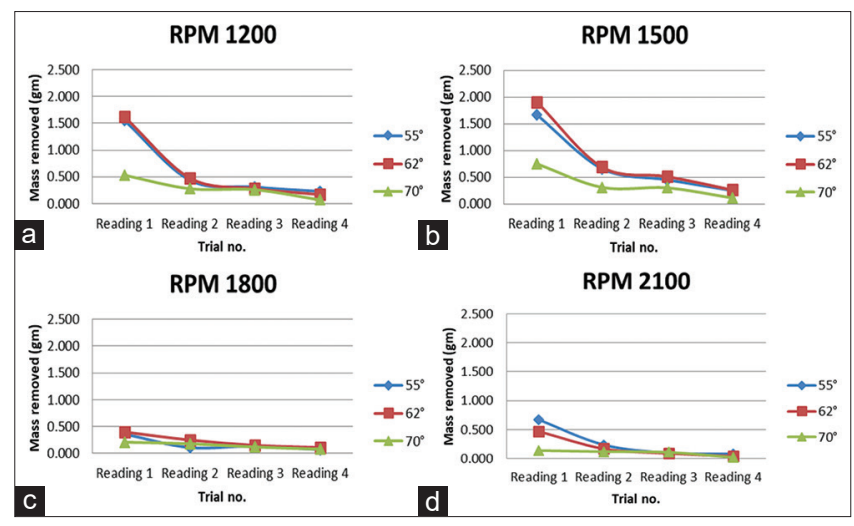

Figure 7: Four millimeter shaver test results indicate reduction of mass removal using the same shaver for several times

\section{Discussion}

The cutting tip should be used carefully to minimize the risk of unintended tissue or arthroscope damage. The cutting process has to be smooth that the shaver should not be forced to produce distraction. Adequate joint space or distension is necessary during arthroscopic inspection and arthroscopic shaver use. Shavers should be used cautious cutting in patients with stiff joints or ankylosis [4].

Tooth point angles $55^{\circ}$ and $62^{\circ}$ showed the highest removal rates whatever the shaver diameter and cutting speed are. That increasing tooth point angle may reduce tissue removal rate, this finding matched theoretical studied that may be referred to tooth sharpness. The trend of shaver tests followed and confirmed the validity of the FE model [6], [7] previously conducted on ANSYS that resulted in suggesting that the optimal range for shaver teeth should be between

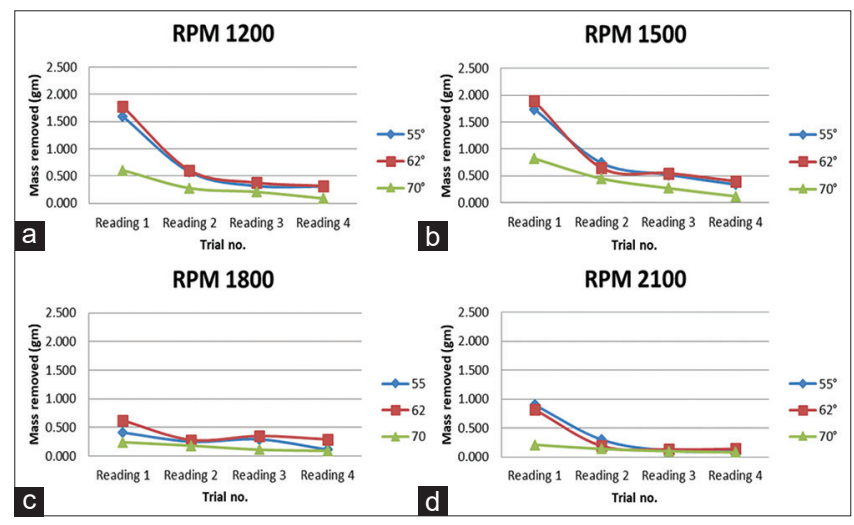

Figure 8: Six millimeters shaver test results indicate reduction of mass removal using the same shaver for several times $55^{\circ}$ and $62^{\circ}$ from the stresses experienced point of view.

Increasing rotating speed to $1800 \mathrm{rpm}$ and more may cause sliding of cutting teeth. On the other hand, the theoretical studies recommended shaving operation at around $1500 \mathrm{rpm}$ [6], [7] for better performance (maximum removal rate) that was proved experimentally within this study. Arthroscopic system has three working modes including counter-wise, counter-clockwise, and oscillation. Its rotating speed is about $100-10,000 \mathrm{rpm}$. Compared to the other two working modes, the cutting rate of shaver in oscillation rotation modes is higher [11].

Wieser et al. [12] tested three shaver systems and blades; (1) the Karl Storz Power Shaver S2 (Karl Storz, Tuttlingen, Germany), (2) the Stryker CORE Shaver System (Stryker, Kalamazoo, MI), and (3) the Dyonics Power Shaver System (Smith and Nephew, Andover, MA) to find out best operating conditions. The optimal revolutions per minute for maximal resection performance at $5 \mathrm{~N}$ were recorded. For the Karl Storz and Stryker systems, the best performance was obtained with approximately $2500 \mathrm{rpm}$. On the other hand, the Smith and Nephew system provided its best performance at $1500 \mathrm{rpm}$. However, with the increase in speed, this resulted in less resection of tissue [12]. The obtained finding within this study matched conclusions by Wieser et al., [12]

Results of microscopic images showed less damage to the sharp teeth of the shavers at the same teeth point angles $\left(55^{\circ}\right.$ and $\left.62^{\circ}\right)$. The trend of the removal rate curves could be interpreted as having a certain range of angles where smaller angles would result in more penetrating than shearing, while larger angles result in more shearing than penetrating. Thus, a compromise for both is made to achieve acceptable results.

\section{Conclusions}

Three shaver designs (three teeth point angles: $55^{\circ}, 62^{\circ}$, and $70^{\circ}$ ) were manufactured on two diameters $4 \mathrm{~mm}$ and $6 \mathrm{~mm}$. Within this study, these shavers were tested on bovine articular cartilage specimens with four different cutting speeds on four consecutive cycles, as well as controlling the contact force. In addition to test rig designing and performing shavers tests in a controlled laboratory environment, this research can be considered as the experimental validation of the previous FE model results [6], [7].

Results of the experimental work validated and confirmed the results of the FE simulation previously conducted that found cutting speed of $1500 \mathrm{rpm}(14 \mathrm{~m} / \mathrm{min})$ achieves the highest resection 
rate for all the shaver designs tested (approximately $60 \%$ higher).

In addition, confirming that the shavers of teeth angles between $55^{\circ}$ and $62^{\circ}$ would experience less teeth damage, hence suggesting their subjection to less stresses while cutting as earlier recommended from the FE model results. Drop in resection performance every 3 min cycle was found to be approximately $50 \%$.

\section{Acknowledgment}

This research was carried out through a project entitled "Redesign of some Endoscopic Instruments and Implants (GIT and joints)." That was funded by the National Research Centre - NRC, internal project (code: 11090336), Egypt.

\section{References}

1. Whipple TL. Powered instruments for wrist arthroscopy. Arthroscopy. 1988;4(4):290-4.

PMid:3233120

2. Phillips BB. General principles of arthroscopy. In: Canale ST, editor. Campbell's Operative Orthopaedics. $10^{\text {th }}$ ed. Maryland Heights, Missouri: Mosby-Year Book Inc.; 2002. p. 1463-9.
3. Dandy D. Arthroscopic Management of the Knee. $2^{\text {nd }}$ ed. London, United Kingdom: Churchill Livingstone; 1987. p. 23-7.

4. Singh S, Tavakkolizadeh A, Arya A, Compson J. Arthroscopic powered instruments: Areview of shavers and burrs. Orthop Trauma. 2009;23(5):357-61. https://doi.org/10.1016/j.mporth.2009.01.002

5. Horeman T, Schilder F, Aguirre M, Kerkhoffs G, Tuijthof G. Design and preliminary evaluation of a stiff steerable cutter for arthroscopic procedures. J Med Devices. 2015;9(4):044503. https://doi.org/10.1115/1.4030506

6. Hafez N, El-Anwar MI, Atia MR. Enhancing the design of arthroscopic shaver to reduce stresses experienced. J Phys Conf Ser. 2020;1447(1):012058. https://doi. org/10.1088/1742-6596/1447/1/012058

7. Hafez N, El-Anwar MI, Atia MR. Enhancing the Design of Arthroscopic Shaver to Reduce Stresses Experienced. Cairo, Egypt: The $4^{\text {th }}$ International Conference on Advanced Technology and Applied Sciences, 10-12 Sept; 2019. https:// doi.org/10.1088/1742-6596/1447/1/012058

8. Available from: https://www.itead.cc/hx711-dual-channelweighing-sensor-module.html. [Last accessed on 2020 Dec 01].

9. Available from: https://www.tmt.com.sa/en/Node/d/23. [Last accessed on 2020 Dec 01].

10. Available from: https://www.kern-sohn.com/shop/en/laboratorybalances/precision-balances/PCB. [Last accessed on 2020 Dec 01].

11. Chen Z, Wang C, Jiang W, Tang N, Chen B. A review on surgical instruments of knee arthroscopic debridement and total hip arthroplasty. Proc CIRP. 2017;65:291-8. https://doi. org/10.1016/j.procir.2017.05.001

12. Wieser K, Erschbamer M, Neuhofer S, Ek ET, Gerber CC, Meyer DC. Controlled laboratory testing of arthroscopic shaver systems: Do blades, contact pressure, and speed influence their performance? Arthroscopy. 2012;28(10):1497-503. https://doi. org/10.1016/j.arthro.2012.03.006

PMid:22683374 